EPJ Web of Conferences 41, 07009 (2013)

DOI: $10.1051 /$ epjconf/20134107009

(C) Owned by the authors, published by EDP Sciences, 2013

\title{
Transient IR study of Blue Light Sensing Proteins
}

\author{
A. Lukacs ${ }^{1,4}$, A. Haigney ${ }^{2}$, R. Brust ${ }^{2}$, R. Zhao ${ }^{1}$, G. Greetham ${ }^{3}$, M. Towrie ${ }^{3}$, P.J. Tonge ${ }^{2}$, S.R. \\ Meech $^{1}$ \\ ${ }^{1}$ University of East Anglia, School of Chemistry, Norwich, UK \\ ${ }^{2}$ Stony Brook University, Department of Chemistry, Stony Brook, New York, USA \\ ${ }^{3}$ Central Laser Facility, Rutherford Appleton Laboratory, Didcot, UK \\ ${ }^{4}$ University of Pecs, Medical School, Department of Biophysics, Hungary
}

\begin{abstract}
In this work we present the identification of redox intermediates of flavin and tryptophan after photoexcitation in glucose oxidase, Y21W AppA mutant and 1AppA by the means of TRIR spectroscopy.
\end{abstract}

\section{Introduction}

Redox properties of flavin molecules endow flavoenzymes with a huge versatility in their function. Their ability to exist in several redox states means that they can take part in a wide variety of redox processes[1]. Photoactive flavoproteins are a special class of protein where the blue light absorbing flavin (FAD, FMN) cofactor plays a role in a light activated function. An important class of photoactive flavoproteins are the Blue Light Using FAD (BLUF) domain family involved in the photophobic response and in light controlled gene expression in bacteria [2]. The mechanism of photoactivity in the BLUF domain has not yet been definitively established. Detailed studies of the gene-regulating AppA protein, which contains one of the best characterised BLUF domains [3-5] reveal a kinetically complex photocycle with a number of steps occurring on the picosecond time scale. However transient visible and mid IR spectroscopy has yet to identify any intermediates between the dark adapted state and the light activated signalling state.

In this work we present ultrafast transient absorption measurements in the visible and mid-infrared region performed on GOX (where FAD was in oxidized and neutral semiquinone state), AppA and the AppA mutant Y21W. With these methods we are able to identify specific spectroscopic signatures of potential flavin and tryptophan redox intermediates in various photoactive flavoproteins, and to record their formation and decay kinetics.

\section{Experimental methods}

Time resolved infrared measurements were performed on an ultrafast laser system with a $10 \mathrm{kHz}$ repetition rate and $\sim 100 \mathrm{fs}$ time resolution. For these measurements the excitation wavelength was $400 \mathrm{~nm}$, with a spot size radius of $\sim 100 \mu \mathrm{m}$. The pulse energy was kept below $400 \mathrm{~nJ}$ per pulse. Photoactive proteins were studied in a micro flow cell $(0.5 \mathrm{ml}$ volume total, $50 \mu \mathrm{m}$ path length $)$ with $\mathrm{CaF}_{2}$ windows, which was rastered in the beam path, thus avoiding photobleaching and degradation of the proteins during the measurement. The pump probe polarization angle was set to magic angle 
to eliminate any effects of molecular reorientation. The infrared spectra were measured in the $\sim 1400$ $1800 \mathrm{~cm}^{-1}$ region by two carefully matched 128 pixel MCT detectors, yielding a resolution of $3 \mathrm{~cm}^{-1}$ per pixel.

\section{Results and discussion}

Oxidized GOX was measured at several delay times after photoexcitation: the spectrum of the oxidized GOX (Fig. 1) at early times after excitation is very similar to the spectrum of oxidized FAD $[6,7]$. The major ground state bleaches at $1553 \mathrm{~cm}^{-1}$, at $1651 \mathrm{~cm}^{-1}$ and $1703 \mathrm{~cm}^{-1}$ have been assigned to mainly $\mathrm{C} 10 \mathrm{aN} 1, \mathrm{C}_{2}=\mathrm{O}$ and $\mathrm{C}_{4}=\mathrm{O}$ localized vibrations of the isoalloxazine ring, respectively through DFT calculation and isotope substitution [6-8].

We observed a monoexponential fast $\sim 3 \mathrm{ps}$ decay of the excited state at $1611 \mathrm{~cm}^{-1}$ and a heterogeneous ground state recovery at $1550 \mathrm{~cm}^{-1}$ with the time constants $\sim 3$ ps and $\sim 26 \mathrm{ps}$. A very interesting feature was observed at $1628 \mathrm{~cm}^{-1}$ (Fig 1.) where the change of the absorption is decreasing rapidly before the recovery. At $1525 \mathrm{~cm}^{-1}$ one can observe a parallel kinetics, the absorption change increasing rapidly before decay. Knowing that in oxidized GOX after excitation the nearby tryptophan serves as an electron donor we identify this behaviour with the disappearance of the tryptophan mode at $1628 \mathrm{~cm}^{-1}$ and the appearance of the $\mathrm{TrpH}^{\mathrm{o}+}$ at $1525 \mathrm{~cm}^{-1}$.

We observed strikingly similar kinetics in 1AppA (not shown here) but no such kinetics in dAppA, suggesting that any electron transfer step does not involve W104. In 1AppA the closest tryptophan (W104) is in a different position which is unfavourable for the electron transfer. In order to eliminate the possible tyrosine radical intermediates we constructed a mutant $\mathrm{Y} 21 \mathrm{~W}$ where the closest tyrosine (Y21) was exchanged to a tryptophan with the assumption that in the case of electron transfer on might see an enhanced signal for the formation of the tryptophan cation radical.

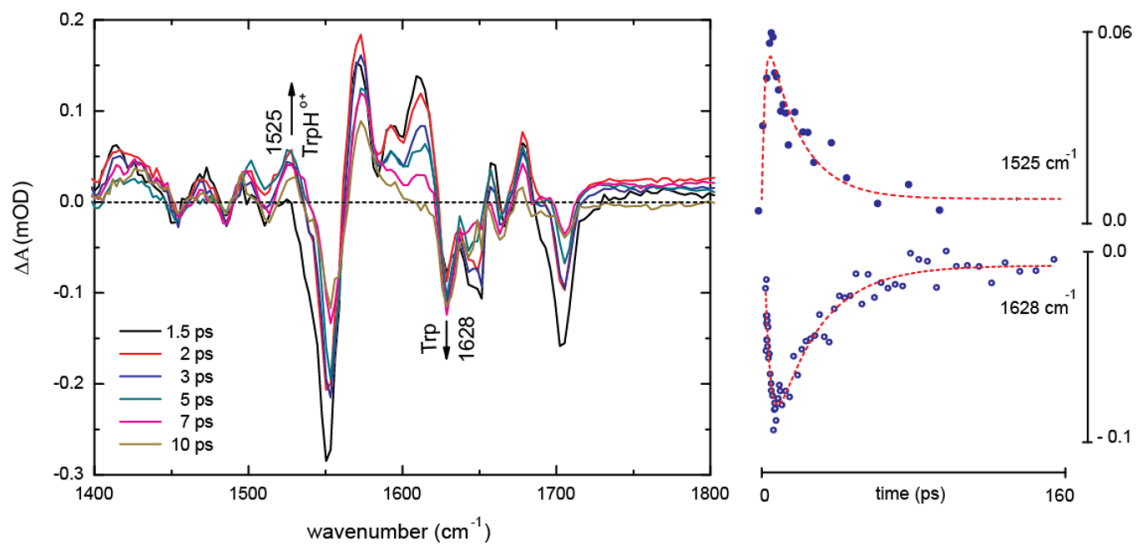

Fig. 1 Oxidized GOX measured at different delay times and the kinetics observed at $1525 \mathrm{~cm}^{-1}$ and $1628 \mathrm{~cm}^{-1}$

The exchange of the tyrosine to a tryptophan resulted in the loss of photoactivity of AppA which is possibly due to the disruption of the hydrogen bonding network around the flavin chromophore in the wild type protein. The lack of photoactivity can be seen in the spectra of the mutant (Fig. 2) as the typical shift of the vibrational mode of FAD in dAppA at $\sim 1703 \mathrm{~cm}^{-1}$ to $\sim 1686 \mathrm{~cm}^{-1}$ in 1 AppA is missing. 


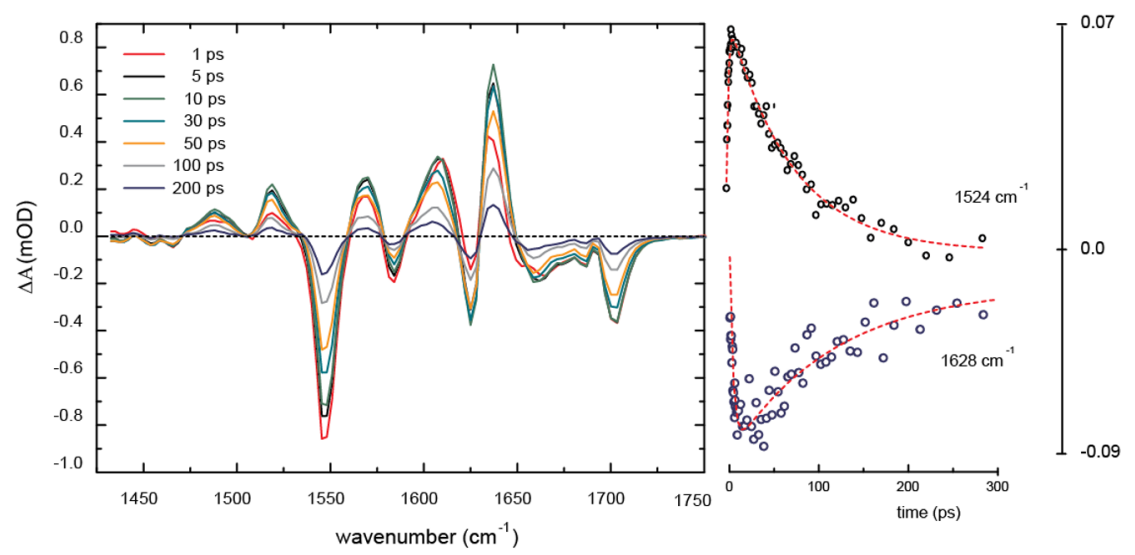

Fig.2 TRIR spectra of Y21W, and the observed kinetics at $1524 \mathrm{~cm}^{-1}$ and $1628 \mathrm{~cm}^{-1}$

The kinetics observed at $1524 \mathrm{~cm}^{-1}$ and $1628 \mathrm{~cm}^{-1}$ is very similar to those which were observed in GOX, the only difference that the second time constant is longer ( $>100 \mathrm{ps})$ than the one observed in GOX. This proves that the transient appearing at $\sim 1525 \mathrm{~cm}^{-1}$ can be identified as the IR marker of $\mathrm{TrpH}^{\mathrm{o}+}$.

\section{Conclusions}

By the means of TRIR we were able to identify the infrared spectrum of $\mathrm{FAD}^{\mathrm{o}-}$ and $\mathrm{TrpH}^{\mathrm{o}+}$ in two flavoprotein, GOX and the transcriptional antirepressor AppA. The identification of these redox intermediates has an important role in the characterization of the different steps of the photocycle in BLUF proteins.

\section{References}

1. Massey, V., The chemical and biological versatility of riboflavin. Biochemical Society Transactions, 2000. 28(4): p. 283-96.

2. Losi, A., Flavin-based blue-light photosensors: A photobiophysics update. Photochemistry and Photobiology, 2007. 83(6): p. 1283-1300.

3. Anderson, S., et al., Structure of a novel photoreceptor, the BLUF domain of AppA from Rhodobacter sphaeroides. Biochemistry, 2005. 44(22): p. 7998-8005.

4. Masuda, S. and C.E. Bauer, AppA is a blue light photoreceptor that antirepresses photosynthesis gene expression in Rhodobacter sphaeroides. Cell, 2002. 110(5): p. 613623.

5. Unno, M., et al., Light-induced structural changes in the active site of the BLUF domain in AppA by Raman spectroscopy. Journal of Physical Chemistry B, 2005. 109(25): p. 1262012626.

6. Kondo, M., et al., Ultrafast vibrational spectroscopy of the flavin chromophore. Journal of Physical Chemistry B, 2006. 110(41): p. 20107-20110.

7. Haigney, A., et al., Ultrafast infrared spectroscopy of an isotope-labeled photoactivatable flavoprotein. Biochemistry, 2011. 50(8): p. 1321-8.

8. Wolf, M.M.N., et al., Ultrafast Infrared Spectroscopy of Riboflavin: Dynamics, Electronic Structure, and Vibrational Mode Analysis. Journal of Physical Chemistry B, 2008. 112(42): p. 13424-13432. 